\title{
Investigation of Adding Proportion of RAP in Recycled Asphalt Mixture
}

\author{
CAO He ${ }^{1,3, a}$, ZHU Hao ${ }^{2 *, b}$, LI Caixia ${ }^{4, c}$, SUN Anshi ${ }^{2, \mathrm{~d}}$, LI Jiange ${ }^{2, \mathrm{e}}$, and LI Shuang ${ }^{2, \mathrm{f}}$ \\ ${ }^{1}$ Guangxi Key Lab of Road Structure and Materials, Nanning, 530007 ,China \\ ${ }^{2}$ Key Laboratory of Road Structure \& Material Ministry of Transport, Chang' an University, Xi' an \\ 710064 ,China \\ ${ }^{3}$ Guangxi Transportation Research Institute, Nanning, 530007, China \\ ${ }^{4}$ Shaanxi College of Communication Technology, Shaanxi, 710018, ${ }^{\text {China }}$ \\ a147866246@qq.com, b1720120436@qq.com,,23806224@qq.com, d1125132337@qq.com, \\ e745097840@qq.com, $1406118621 @ q q . c o m$
}

\begin{abstract}
According to the relationship between gradation and proportion of Reclaimed Asphalt Pavement (RAP) and design gradation of recycled mixture, the authors discussed the influence of proportion of RAP on gradation adjustment of recycled mixture. And then, recycled mixture with $0 \%, 30 \%, 50 \%, 70 \%$ of RAP were made, and Influence of proportion of RAP on high and low temperature performance, water stability and anti-aging performance of recycled mixture were discussed. The results and analysis indicate that gradation of recycled mixture would not be adjusted to aiming gradation if proportion of RAP was too big. With the increase of proportion of RAP, high temperature performance and anti-aging performance of recycled mixture enhanced, but low temperature performance and water stability decayed sharply. In practical application, reasonable proportion of RAP should be determined according to gradation, performance demand and economy of recycled mixture.

Keywords: recycled asphalt mixture; proportion of RAP; gradation; road performance
\end{abstract}

\section{Introduction}

Recycling technology of asphalt pavement is a technology which formed recycled asphalt mixture renew pavement. After through digging, recycling, fragmentation, screening of old asphalt pavement which need renovation or waste, have a mixture with recycling agent, new asphalt material, new aggregate by a certain percentage in the factory or on site ${ }^{[1-4]}$. The proportion of RAP in recycled asphalt mixture influence not only economy, but also the using performance of recycled pavement ${ }^{[3-4]}$. As we all know, the higher of proportion of RAP is, the more economic social benefits, but if the proportion of RAP is too high will result the road performance of recycled mixture poor, even failure. Data shows that the proportion of RAP in recycled mixture generally is $30-50 \%$, but it usually depends on experience and

*Corresponding author: 1720120436@qq.com 
lack of necessary theoretical guidance. In practice, firstly determine the proportion of RAP and then determine the dosage of new aggregate, new asphalt and recycling agent. So it's necessary to study the effect of proportion of RAP on recycled asphalt mixture about gradation design and road performance.

\section{Influence of Proportion of RAP on Gradation of Recycled Mixture}

The recycle of aggregate is put fresh aggregate into old asphalt mixture to readjust the gradation of mixture for meet the requirement of the prescriptive gradation. In this research, according to the relationship between gradation, proportion of RAP and design gradation of recycled asphalt mixture, a compound formula of old and new aggregate were raised.

$$
N_{i}=\left(M_{i}-P_{i} \times a\right) /(1-a)
$$

Where, $P_{i}$ - the residual amount on each sieve of RAP $/ \%$ ( $i$ is the size of each sieve pore); $M_{l}$ - the residual amount on each sieve designed/\%; a - the proportion of RAP ); $N_{l}$ - the residual amount on each sieve of fresh aggregate which need to be added.

When proportion of RAP is not too large, $N_{i}$ is positive, and the gradation of the mixture of old material and new material is consistent with aiming gradation. While if the proportion of RAP is too big, $N_{i}$ maybe negative. This shows that in this proportion, RAP would not be fully adjusted to design gradation. For example, when the proportion of RAP is $50 \%$, the numerical of $N_{i}$ multi-range is negative. Tablel shows that in the design of recycled mixture if proportion of RAP is too big, it would not be adjusted to the aiming gradation, so the proportion of RAP should be reduced appropriately and recalculate.

TABLE 1 INFLUENCE OF PROPORTION OF RAP ON ADDED PROPORTION OF FRESH AGGREGATE

\begin{tabular}{cccccc}
\hline Sieve size $/(\mathrm{mm})$ & $M_{i} /(\%)$ & $P_{i} /(\%)$ & $N_{i} / \%$ & $(\mathrm{a}=35 \%)$ & $N_{i}(\%)$ \\
\hline 16 & 5 & 2.4 & 6.4 & 7.6 \\
13.2 & 11 & 4.6 & 14.4 & 17.4 \\
9.5 & 13.5 & 8.1 & 16.4 & 18.9 \\
4.75 & 23.5 & 29 & 20.5 & 18.0 \\
2.36 & 15 & 20.5 & 12.0 & 9.5 \\
1.18 & 11.5 & 7.8 & 13.5 & 15.2 \\
0.6 & 6 & 5 & 6.5 & 7.0 \\
0.3 & 3.5 & 9.3 & 0.4 & -2.3 \\
0.15 & 2.5 & 3.4 & 2.0 & 1.6 \\
0.075 & 3.5 & 3.7 & 3.4 & 3.3 \\
$<0.075$ & 5 & 6.2 & 4.4 & 3.8 \\
\hline
\end{tabular}

\section{Effect of Proportion of RAP on Performance of Recycled Mixture}

Recycled mixture with $0 \%, 30 \%, 50 \%, 70 \%$ of RAP were designed and prepared. The limestone and Xishihua- 90 asphalt were used for new aggregate and binder respectively. For the recycle of waste asphalt mixture, whether it needs recycling agent depends on aging degree of asphalt. To make a contrast, the performances of recycled asphalt mixture with/without recycling agent were compared. Due to the use of $70 \%$ of proportion would not adjusted to the same aiming gradation with $30 \%$ and $50 \%$, so gradation was slightly adjusted, used gradation was shown in Table. 2 . 
TABLE 2 AGGREGATE GRADATION

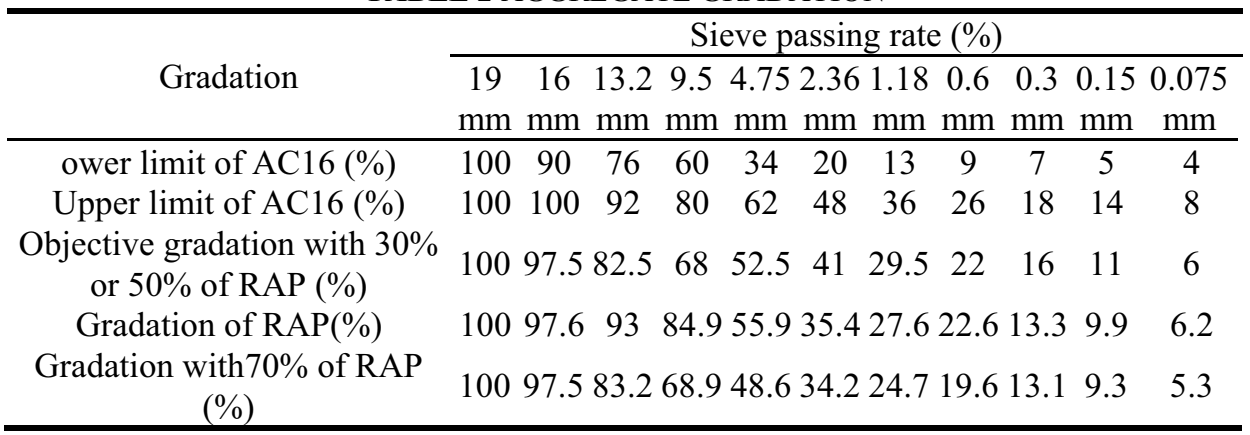

\section{Effect of Proportion of RAP on High Temperature Performance}

According to the specification ${ }^{[8]}$, rutting test of recycled asphalt mixture was conducted. The dynamic stability and deformation in time of 60min were shown in Fig. 1.

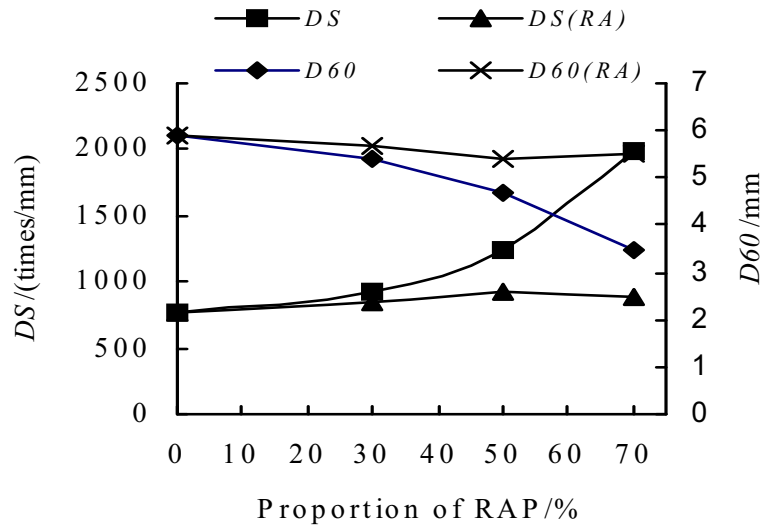

Fig.1 Results of rutting test

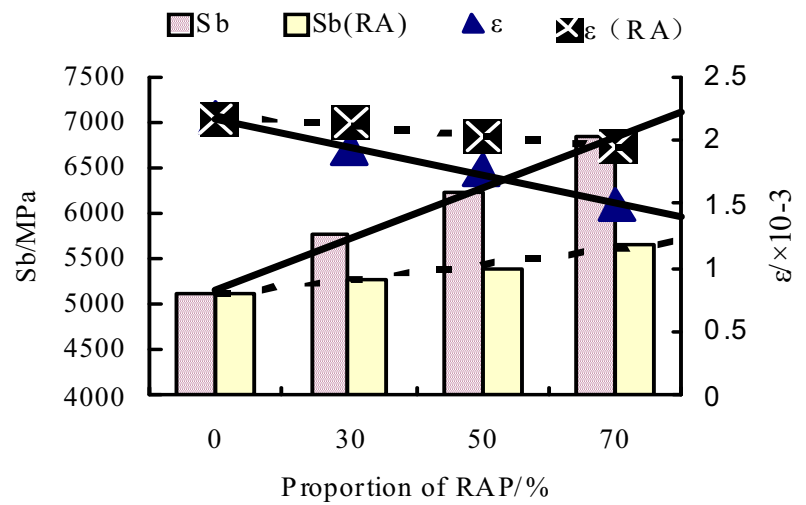

Fig. 2 Test results of low-temperature bending

It can be seen from Fig.1, the dynamic stability (DS) of recycled mixture without recycling agent continued to increase as the proportion of RAP increased. Among them, the DS of mixture with $30 \%$ proportion of RAP increased by $22.1 \%$ than fresh materials , the DS 
of mixture with $50 \%$ proportion of RAP increased by $62.8 \%$ than fresh materials, the DS of mixture with $70 \%$ proportion of RAP increased by $159.7 \%$ than fresh materials. However, rutting deformation in time of 60min gradually decreased as the proportion of RAP increased. The reason is that the viscosity and stiffness of asphalt in RAP increased because of ageing effect. High temperature performance of recycled mixture gradually increased as proportion of RAP increased.

With the increase in proportion of RAP, the DS of recycled mixture with recycling agent has no significant increase, the deformation at $60 \mathrm{~min}(D 60)$ has no significant decrease, and these two indexes have little difference compared to new asphalt mixture. For the same proportion of RAP, the high temperature performance of recycled mixture with recycling agent is less than that of the recycled mixture without recycling agent. The reason is that the addition of recycling agent can soften aged asphalt in RAP and reduce viscosity. The addition quantity of recycling agent was according to the proportion of aged asphalt content in RAP added, so along with the increase of proportion of RAP, high temperature performance of recycled mixture changed significantly has not happened.

\section{Effect of Proportion of RAP on Low Temperature Performance}

Recycled asphalt mixture contains aged asphalt mixture, and the asphalt has experienced a long time of aging, viscosity and stiffness is large, low temperature performance greatly reduced $^{[9]}$. Although recycling agent was added to adjust it, but maybe due to mixing and permanent insufficiently, result in bad regeneration, so that low temperature performance of recycled mixture was affected. So the low temperature performance of recycled asphalt mixture should be the focus on. In low-temperature bending test, determining maximum load and mid-span deflection at failure, through calculate bending strain energy of mixture to evaluate low temperature performance of asphalt mixture.

From Fig.2, we can see that maximum flexure tensile strain near linear decrease, and bending stiffness modulus $(S b)$ near linear increase with the increase in proportion of RAP for recycled asphalt mixture without recycling agent. Maximum flexure tensile strain $(\varepsilon)$ with $70 \%$ proportion recycled material of RAP decreased by $31.6 \%$, bending stiffness modulus increased by $33.7 \%$. And the low temperature deformation abilities of recycled mixture decreased rapidly with the increase of proportion, recycled pavement which use the larger proportion of RAP has larger possibility of cracking, it also explains that the greatest dangers of asphalt pavement aging depends on largely attenuate of low temperature performance.

For recycled mixture with recycling agent, with the increase of proportion of RAP, maximum flexure tensile strain and bending stiffness modulus also close to linear changes, but slope change is very small, change range is obviously smaller than recycled mixture without recycling agent. This sufficiently indicates that adding recycling agent can effectively improve low temperature ductility of asphalt. For recycled asphalt mixture with large proportion, appropriate amount of recycling agent should added to recover low temperature performance.

\section{Effect of Proportion of RAP on Water Stability}

The oxidation aging of asphalt generate some polar compounds, such as sulfonic acid and surfactant, they have strong hydrophilicity, which will aggravate water damage of mixture ${ }^{[10]}$. Recycled asphalt mixture contains aged asphalt, the water damage resistance maybe attenuated, so evaluate the water stability is very important. Immersion Marshall test and freeze-thaw split test were used to study the effect of aging to water stability of asphalt 
mixture. The immersion residual stability and freeze-thaw split strength ratio with different proportion of RAP is shown in Fig.3.

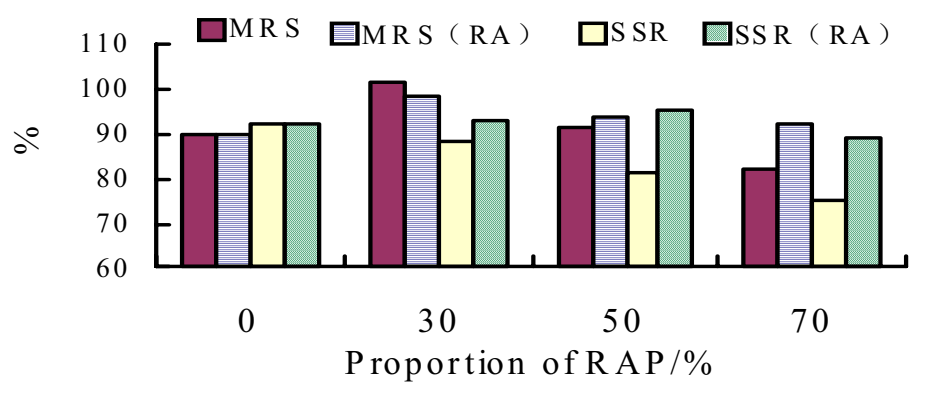

Fig. 3 Results of immersion Marshall test and freeze-thaw split test

Fig. 3 shows that the change law of immersion Marshall residual stability (MRS) of recycled mixture is not obvious with the change of proportion of RAP. From the index, when the proportion of RAP is $70 \%$, water stability is poor; when the proportion of RAP is $50 \%$ and $30 \%$, the water stability show less difference compared with fresh material, and when the proportion of RAP is $30 \%$, immersion residual stability even more than $100 \%$. This also shows that there are some limitations in using immersion residual stability to evaluate water stability of asphalt mixture.

With the increase of proportion of RAP, the freeze-thaw split strength ratio (SSR) of recycled mixture without recycling agent decreased gradually, the proportion of RAP has great effect on water stability of recycled mixture. However, for recycled mixture with the same proportion of RAP, the freeze-thaw split strength ratio of recycled mixture with recycling agent increased significantly.

\section{Effect of Proportion of RAP on Anti-aging Performance}

The anti-aging performance of asphalt mixture is an important index of asphalt pavement durability. For recycled asphalt mixture, durability is the key to measure the success of recycling of waste asphalt mixture. According to Standard Test Methods of Bitumen and Bituminous Mixtures for Highway Engineering, make short-term aging of mixture in oven at $135^{\circ} \mathrm{C}$ for $2 \mathrm{~h}$ and then compaction molding, put compaction samples into separator of Strong wind furnace at $85 \pm 1{ }^{\circ} \mathrm{C}$ for $120 \pm 0.5 \mathrm{~h}$, to simulate long-term aging about $6 \sim 9$ years. Using index change of recycled mixture before and after aging about high temperature rutting test and low temperature bending test to evaluate anti-aging performance of recycled asphalt mixture, the results is shown in Fig.4. 


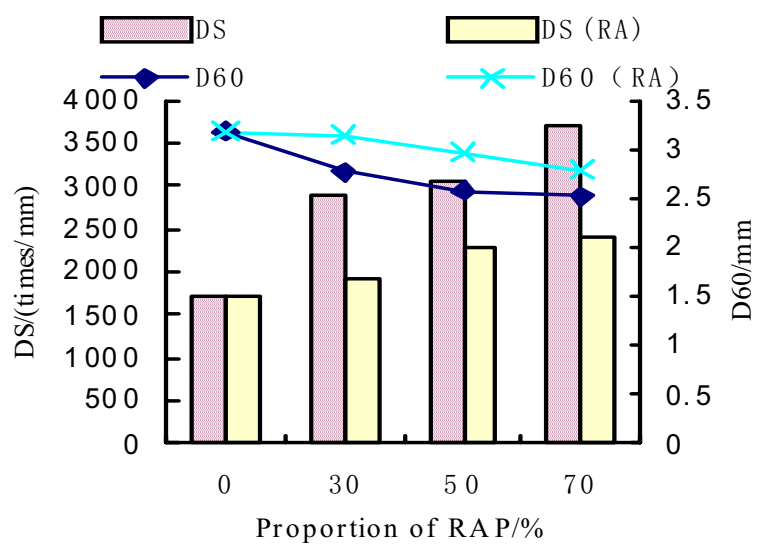

(a) rutting test

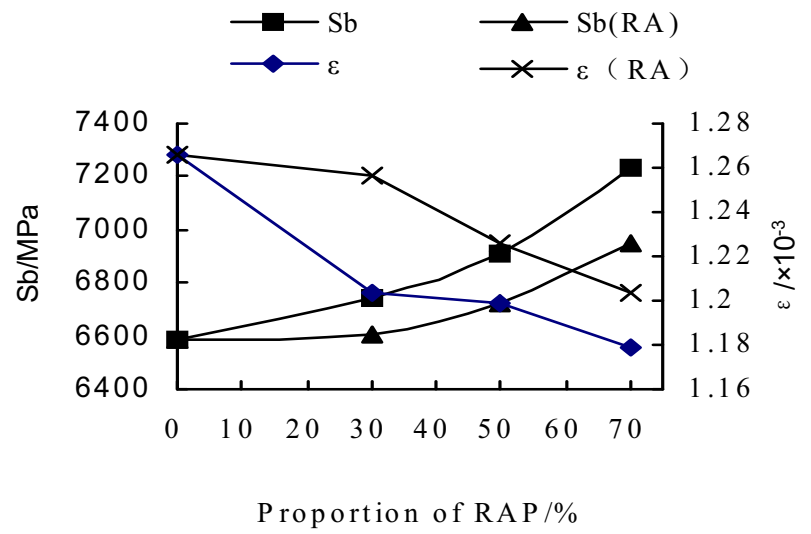

(b) low-temperature bending test

Fig. 4 The performance changes of recycled asphalt mixture before and after aging

From Fig. 4 we can see that $D S$ of recycled mixture after aging both increased, while permanent deformation both decreased, this is consistent with new material whether adding recycling agent or not. But adding recycling agent delayed the change trend obviously, that is, adding recycling agent can enhance anti-aging performance of recycled mixture and delay aging process of recycled pavement.

After aging the low-temperature performance of various mixtures has different degrees of attenuation, among them the index change range of new material is the largest, the bigger proportion of RAP without recycling agent, the smaller change ranges. For recycled material without recycling agent, along with the increase of proportion of RAP, after aging low temperature stiffness gradually increased, however maximal flexural-tensile strain gradually decreased. Although mixture with recycling agent after aging index change range increased, but low temperature performance increased significantly. Consistent with the rutting index is the additive of recycling agent delayed this changing trend, that is to say, after adding recycled agent enhanced anti-aging performance. 


\section{Summary}

(1) If proportion of RAP is too big, it maybe would not adjust to the aiming gradation. So in practical application, reasonable proportion of RAP should be determined according to gradation, performance demand and economy of recycled mixture.

(2) Along with the increase of proportion of RAP, the high temperature performance of recycled asphalt mixture without recycling agent gradually increased, low temperature performance and water stability gradually decreased. However, for recycled mixture using recycling agent, the high temperature performance of recycled mixture with different proportion of RAP without obvious difference, low temperature performance and water stability were all attenuated, but decay rate significantly lower than recycled mixture at the same proportion of RAP which without recycling agent.

(3) Along with the increase of proportion of RAP, high and low temperature performance of recycled asphalt mixture after aging index change range decreased. So whether it good or bad of recycled mixture about anti-aging performance would not just form index change range to evaluate, we should consider the size of the actual performance index after aging of recycled mixture.

\section{Acknowledgments}

This work is supported by the Open Fund of Guangxi Key Lab of Road Structure and Materials (2014gxjgclkf-001), Applied basic research project the ministry of transport of China (No. 2014319812151) and Natural Science Basic Research Plan in Shaanxi Province of China (No. 2014JQ7242). The authors gratefully acknowledge their financial support.

\section{References}

1. S. Paranavithana and A. Mohajerani, "Effects of recycled concrete aggregates on properties of asphalt concrete,"Resources, Conservation and Recycling, 2006, vol.48,no.1,pp.1-12

2. Shen, D. H. and Du, J. C., "Evaluation of Building Materials Recycling on HMA Permanent Deformation,"Constr. Build. Mater., Vol. 18, 2004, pp. 391-397.

3. Yang Jian-ming, Yang Shi-jiao, Xiong Shao-feng, et al. Technology and Actualities of Recycled Mixture for Old Asphalt Pavement. Journal of Nanhua University(Science \& Engineering Edition), 2003, 17 (1) : 11-15

4. Wei Qin, Yang Chang-hui, Xiong Chu-hua, et al. Recycl ing Technology of Aged Asphalt Pavement. Journal of Chongqing Jianzhu University, 2007, 29 (3) : 128-131

5. Huang Xiao-ming, Zhao Yong-li, Jiang Chen. Test and analysis of recycled mixture for old asphalt pavement. Chinese Journal of Geotechnical Engineering, 2001,23(4): 468-471

6. Shen Guo-yin. Test and Analysis of Recycled Mixture for Old Pavement. Highway, 2003, (5): 107-110

7. Jiang Li, Liu Yu-lei. Recycle of waste asphalt mixture. Journal of Northeast Forestry University, 2007, 35 (2) : 88-89

8. Ministry of Communications. Highway asphalt and asphalt mixture testing procedures [S]. Beijing: China Communications Press, 2000

9. Mills-Beale, J. and You, Z., "The Mechanical Properties of Asphalt Mixtures With Recycled Concrete Aggregates," Constr. Build. Mater., Vol. 24, 2010, pp. 230-235.

10. Rafi M. M., Qadir A., Ali S., Siddiqui S. H. Performance of Hot Mix Asphalt Mixtures Made of Recycled Aggregates. Journal of Testing and Evaluation, 2014, 42(2):1-10 